\title{
Global Gronwall Estimates for Integral Curves on Riemannian Manifolds
}

\author{
Michael KUNZINGER, Hermann SCHICHL, \\ Roland Steinbauer, and James A. ViCKERS \\ Fakultät für Mathematik \\ Universität Wien \\ Fakultät für Mathematik \\ Nordbergstrasse 15 \\ Universität Wien \\ 1090 Wien - Austria \\ Michael.Kunzinger@univie.ac .at \\ Nordbergstrasse 15 \\ 1090 Wien - Austria \\ Hermann. Schichl@univie.ac.at \\ Fakultät für Mathematik \\ Universität Wien \\ School of Mathematics \\ University of Southampton \\ Nordbergstrasse 15 \\ 1090 Wien - Austria \\ Highfield, Southampton SO 17 1BJ - UK \\ J.A.Vickers@maths.soton.ac.uk \\ Roland.Steinbauer@univie.ac .at
}

Received: June 13, 2005

Accepted: July 26, 2005

\begin{abstract}
We prove Gronwall-type estimates for the distance of integral curves of smooth vector fields on a Riemannian manifold. Such estimates are of central importance for all methods of solving ODEs in a verified way, i.e., with full control of roundoff errors. Our results may therefore be seen as a prerequisite for the generalization of such methods to the setting of Riemannian manifolds.

Key words: Riemannian geometry, ordinary differential equations, Gronwall estimate. 2000 Mathematics Subject Classification: 53B21, 53C22, 34A26.
\end{abstract}

\section{Introduction}

Suppose that $X$ is a complete smooth vector field on $\mathbb{R}^{n}$, let $p_{0}, q_{0} \in \mathbb{R}^{n}$ and denote by $p(t), q(t)$ the integral curves of $X$ with initial values $p_{0}$ resp. $q_{0}$. In the theory of

Partially supported by the Austrian Science Fund Projects P16742-N04 and START-Project Y237-N13.

Rev. Mat. Complut.

19 (2006), no. 1, 133-137

133

ISSN: $1139-1138$

http://dx.doi.org/10.5209/rev_REMA.2006.v19.n1.16639 
ordinary differential equations it is a well known consequence of Gronwall's inequality that in this situation we have

$$
|p(t)-q(t)| \leq\left|p_{0}-q_{0}\right| e^{C_{T} t} \quad(t \in[0, T))
$$

with $C_{T}=\|D X\|_{L^{\infty}\left(K_{T}\right)}\left(K_{T}\right.$ some compact convex set containing the integral curves $t \mapsto p(t)$ and $t \mapsto q(t))$ and $D X$ the Jacobian of $X$ (cf., e.g., $[1,10.5]$ ).

The aim of this paper is to derive estimates analogous to (1) for integral curves of vector fields on Riemannian manifolds. Apart from a purely analytical interest in this generalization, we note that Gronwall-type estimates play an essential role in the convergence analysis of numerical methods for solving ordinary differential equations (cf. [5]). Concerning notation and terminology from Riemannian geometry our basic references are [2-4].

\section{Estimates}

The following proposition provides the main technical ingredient for the proofs of our Gronwall estimates. Here and in what follows, for $X \in \mathfrak{X}(M)$ (the space of smooth vector fields on $M)$ we denote by $\nabla X$ its covariant differential and by $\|\nabla X(p)\|_{g}$ the mapping norm of $\nabla X(p):\left(T_{p} M,\|\cdot\|_{g}\right) \rightarrow\left(T_{p} M,\|\cdot\|_{g}\right), Y_{p} \mapsto \nabla_{Y_{p}} X$.

Proposition 1.1. Let $[a, b] \ni \tau \mapsto c_{0}(\tau)=: c(0, \tau)$ be a smooth regular curve in $a$ Riemannian manifold $(M, g)$, let $X \in \mathfrak{X}(M)$ and set $c(t, \tau):=\mathrm{Fl}_{t}^{X} c(0, \tau)$ where $\mathrm{Fl}^{X}$ $i s$ the flow of $X$. Choose $T>0$ such that $\mathrm{Fl}^{X}$ is defined on $[0, T] \times c_{0}([a, b])$. Then denoting by $l(t)$ the length of $\tau \mapsto c(t, \tau)$, we have

$$
l(t) \leq l(0) e^{C_{T} t} \quad(t \in[0, T])
$$

where $C_{T}=\sup \left\{\|\nabla X(p)\|_{g}: p \in c([0, T] \times[a, b])\right\}$.

Proof. Let $\tau \mapsto c(0, \tau)$ be parameterized by arc length, $\tau \in[0, l(0)]$. Since $\mathrm{Fl}_{t}^{X}$ is a local diffeomorphism, $g\left(\partial_{\tau} c, \partial_{\tau} c\right)>0$ on $[0, T] \times[a, b]$. Furthermore, since the LeviCività connection $\nabla$ is torsion free, we have $\nabla_{\partial_{t}} c_{\tau}=\nabla_{\partial_{\tau}} c_{t}$, where $c_{t}=\partial_{t} c, c_{\tau}=\partial_{\tau} c$, see $[3,1.8 .14]$. Then

$$
\begin{aligned}
l(s)-l(0) & =\int_{0}^{s} \partial_{t} l(t) d t=\int_{0}^{s} \partial_{t} \int_{0}^{l(0)}\left\|c_{\tau}(t, \tau)\right\|_{g} d \tau d t \\
& =\int_{0}^{s} \int_{0}^{l(0)} \frac{\partial_{t} g\left(c_{\tau}(t, \tau), c_{\tau}(t, \tau)\right)}{2\left\|c_{\tau}(t, \tau)\right\|_{g}} d \tau d t=\int_{0}^{s} \int_{0}^{l(0)} \frac{g\left(\left(\nabla_{\partial_{t}} c_{\tau}\right)(t, \tau), c_{\tau}(t, \tau)\right)}{\left\|c_{\tau}(t, \tau)\right\|_{g}} d \tau d t \\
& =\int_{0}^{s} \int_{0}^{l(0)} \frac{g\left(\left(\nabla_{\partial_{\tau}} c_{t}\right)(t, \tau), c_{\tau}(t, \tau)\right)}{\left\|c_{\tau}(t, \tau)\right\|_{g}} d \tau d t \leq \int_{0}^{s} \int_{0}^{l(0)}\left\|\left(\nabla_{\partial_{\tau}} c_{t}\right)(t, \tau)\right\|_{g} d \tau d t
\end{aligned}
$$




$$
\begin{aligned}
& =\int_{0}^{s} \int_{0}^{l(0)}\left\|\nabla_{c_{\tau}(t, \tau)} X\right\|_{g} d \tau d t \leq C_{T} \int_{0}^{s} \int_{0}^{l(0)}\left\|c_{\tau}(t, \tau)\right\|_{g} d \tau d t \\
& =C_{T} \int_{0}^{s} l(t) d t .
\end{aligned}
$$

The claim now follows by applying Gronwall's inequality.

We may utilize this proposition to prove our first main result:

Theorem 1.2. Let $(M, g)$ be a connected smooth Riemannian manifold, $X \in \mathfrak{X}(M)$ a complete vector field on $M$ and let $p_{0}, q_{0} \in M$. Let $p(t)=\mathrm{Fl}_{t}^{X}\left(p_{0}\right), q(t)=\mathrm{Fl}_{t}^{X}\left(q_{0}\right)$ and suppose that $C:=\sup _{p \in M}\|\nabla X(p)\|_{g}<\infty$. Then

$$
d(p(t), q(t)) \leq d\left(p_{0}, q_{0}\right) e^{C t} \quad(t \in[0, \infty)),
$$

where $d(p, q)$ denotes Riemannian distance.

Proof. For any given $\varepsilon>0$, choose a piecewise smooth regular curve $\tau \mapsto c_{0}(\tau)=$ : $c(0, \tau):[0,1] \rightarrow M$ connecting $p_{0}$ and $q_{0}$ such that $d\left(p_{0}, q_{0}\right)>l(0)-\varepsilon$. Using the notation of Proposition 1.1 it follows that

$$
d(p(t), q(t)) \leq l(t) \leq l(0) e^{C t}<\left(d\left(p_{0}, q_{0}\right)+\varepsilon\right) e^{C t}
$$

for $t \in[0, \infty)$. Since $\varepsilon>0$ was arbitrary, the result follows.

Example 1.3. (i) In general, when neither $M$ nor $X$ is complete, the conclusion of Theorem 1.2 is no longer valid:

Consider $M=\mathbb{R}^{2} \backslash\{(0, y) \mid y \geq 0\}$, endowed with the standard Euclidean metric. Let $X \equiv(0,1), p_{0}=\left(-x_{0},-y_{0}\right)$, and $q_{0}=\left(x_{0},-y_{0}\right)\left(x_{0}>0, y_{0} \geq 0\right)$ (cf. figure 1). Then $p(t)=\left(-x_{0},-y_{0}+t\right), q(t)=\left(x_{0},-y_{0}+t\right)$ and

$$
d(p(t), q(t))= \begin{cases}2 x_{0}, & t \leq y_{0} \\ 2 \sqrt{x_{0}^{2}+\left(t-y_{0}\right)^{2}}, & t>y_{0}\end{cases}
$$

On the other hand, $\nabla X=0$, so (3) is violated for $t>y_{0}$, i.e., as soon as the two trajectories are separated by the "gap" $\{(0, y) \mid y \geq 0\}$.

(ii) Replace $X$ in (i) by the complete vector field $\left(0, e^{-1 / x^{2}+1}\right)$ and set $x_{0}=1$, $y_{0}=0$. Then $C:=\|\nabla X\|_{L^{\infty}\left(\mathbb{R}^{2}\right)}=3 \sqrt{3 /(2 e)}$ and

$$
d(p(t), q(t))=2 \sqrt{1+t^{2}} \leq d\left(p_{0}, q_{0}\right) e^{C t}=2 e^{C t}
$$

for all $t \in[0, \infty)$, in accordance with Theorem 1.2. 


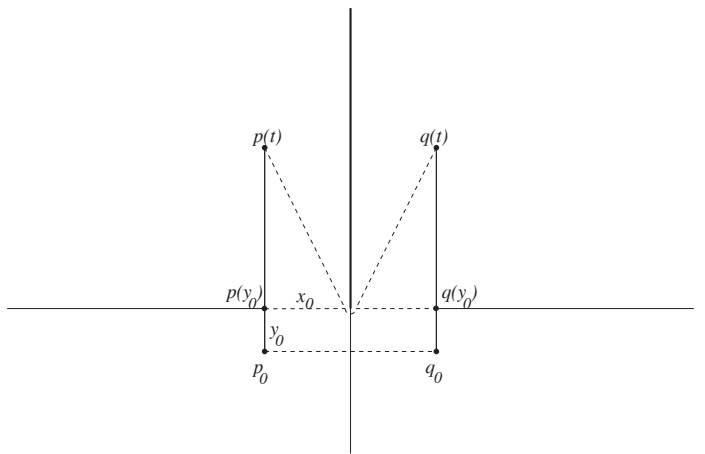

Figure 1

The following result provides a sufficient condition for the validity of a Gronwall estimate even if neither $M$ nor $X$ satisfies a completeness assumption.

Theorem 1.4. Let $(M, g)$ be a connected smooth Riemannian manifold, $X \in \mathfrak{X}(M)$ and let $p_{0}, q_{0} \in M$. Let $p(t)=\mathrm{Fl}_{t}^{X}\left(p_{0}\right), q(t)=\mathrm{Fl}_{t}^{X}\left(q_{0}\right)$ and suppose that there exists some relatively compact submanifold $N$ of $M$ containing $p_{0}, q_{0}$ such that $d\left(p_{0}, q_{0}\right)=$ $d_{N}\left(p_{0}, q_{0}\right)$. Fix $T>0$ such that $\mathrm{Fl}^{X}$ is defined on $[0, T] \times N$ and set $C_{T}:=$ $\sup \left\{\|\nabla X(p)\|_{g}: p \in \mathrm{Fl}^{X}([0, T] \times N)\right\}$. Then

$$
d(p(t), q(t)) \leq d\left(p_{0}, q_{0}\right) e^{C_{T} t} \quad(t \in[0, T]) .
$$

Proof. As in the proof of Theorem 1.2, for any given $\varepsilon>0$ we may choose a piecewise smooth curve $\tau \mapsto c_{0}(\tau):[0,1] \rightarrow N$ from $p_{0}$ to $q_{0}$ such that $d\left(p_{0}, q_{0}\right)=$ $d_{N}\left(p_{0}, q_{0}\right)>l(0)-\varepsilon$. The corresponding time evolutions $c(t, \cdot)$ of $c(0, \cdot)=c_{0}$ then lie in $\mathrm{Fl}^{X}([0, T] \times N)$, so an application of Proposition 1.1 gives the result.

Example 1.5. Clearly such a submanifold $N$ need not exist in general. As a simple example take $M=\mathbb{R}^{2} \backslash\{(0,0)\}, p_{0}=(-1,0), q_{0}=(1,0)$. In Example 1.3.(i) with $y_{0}>0$ the condition is obviously satisfied with $N$ an open neighborhood of the straight line joining $p_{0}, q_{0}$ and the supremum of the maximal evolution times of such $N$ under $\mathrm{Fl}^{X}$ is $T=y_{0}$, coinciding with the maximal time-interval of validity of (4). On the other hand, if there is no $N$ as in Theorem 1.4 then the conclusion in general breaks down even for arbitrarily close initial points $p_{0}, q_{0}$ : if we set $y_{0}=0$ in Example 1.3.(i) then no matter how small $x_{0}$ (i.e., irrespective of the initial distance of the trajectories) the estimate is not valid for any $T>0$.

Finally, we single out some important special cases of Theorem 1.4:

Corollary 1.6. Let $M$ be a connected geodesically complete Riemannian manifold, $X \in \mathfrak{X}(M)$, and $p_{0}, q_{0}, p(t), q(t)$ as above. Let $S$ be a minimizing geodesic segment 
connecting $p_{0}, q_{0}$ and choose some $T>0$ such that $\mathrm{Fl}^{X}$ is defined on $[0, T] \times S$. Then (4) holds with $C_{T}=\sup \left\{\|\nabla X(p)\|_{g} \mid p \in \mathrm{Fl}^{X}([0, T] \times S)\right\}$. In particular, if $X$ is complete then for any $T>0$ we have

$$
d(p(t), q(t)) \leq d\left(p_{0}, q_{0}\right) e^{C_{T} t} \quad(t \in[0, T]) .
$$

Proof. Choose for $N$ in Theorem 1.4 any relatively compact open neighborhood of $S$. The value of $C_{T}$ then follows by continuity.

In particular, for $M=\mathbb{R}^{n}$ with the standard Euclidean metric, Corollary 1.6 reproduces $(1)$.

\section{References}

[1] J. Dieudonné, Foundations of modern analysis, Pure and Applied Mathematics, Academic Press, New York, 1960.

[2] S. Gallot, D. Hulin, and J. Lafontaine, Riemannian geometry, Universitext, Springer-Verlag, Berlin, 2004.

[3] W. P. A. Klingenberg, Riemannian geometry, de Gruyter Studies in Mathematics, vol. 1, Walter de Gruyter \& Co., Berlin, 1995.

[4] B. O'Neill, Semi-Riemannian geometry (with applications to relativity), Pure and Applied Mathematics, vol. 103, Academic Press Inc., New York, 1983.

[5] J. Stoer and R. Bulirsch, Numerische Mathematik 2, Springer-Lehrbuch, Springer-Verlag, Berlin, 1990. 